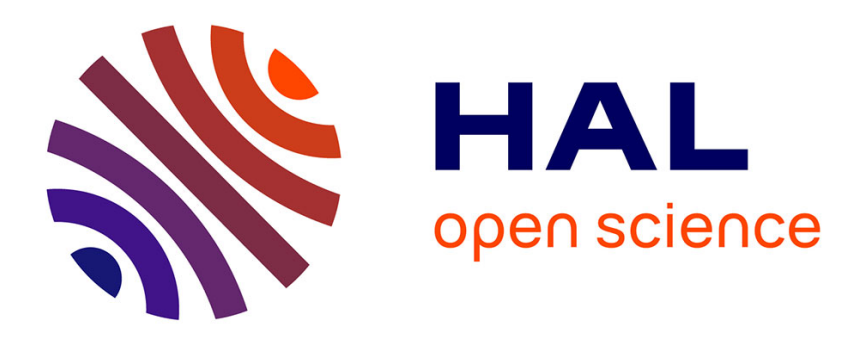

\title{
Thermalization of subexcitation electrons in a medium with time dispersion
}

\author{
S. Stepanov
}

\section{To cite this version:}

S. Stepanov. Thermalization of subexcitation electrons in a medium with time dispersion. Journal de Physique IV Proceedings, 1993, 03 (C4), pp.C4-41-C4-47. 10.1051/jp4:1993404 . jpa-00251447

\section{HAL Id: jpa-00251447 https://hal.science/jpa-00251447}

Submitted on 1 Jan 1993

HAL is a multi-disciplinary open access archive for the deposit and dissemination of scientific research documents, whether they are published or not. The documents may come from teaching and research institutions in France or abroad, or from public or private research centers.
L'archive ouverte pluridisciplinaire HAL, est destinée au dépôt et à la diffusion de documents scientifiques de niveau recherche, publiés ou non, émanant des établissements d'enseignement et de recherche français ou étrangers, des laboratoires publics ou privés. 


\title{
Thermalization of subexcitation electrons in a medium with time dispersion
}

\author{
S.V. STEPANOV
}

Institute of Theoretical and Experimental Physics, 117259 Moscow, Russia

\begin{abstract}
The slowing down process of light charged subexcitation particles of finite size in a medium with time dispersion is considered. The theory developed is applied to the slowing down of the secondary electrons knocked out by another energetic particle. Total energy loss is made up from the Debye losses, excitation of intra- and intermolecular vibrations and the mutual coulomb attraction between parent ion and knocked out lectron. In this case the process is essentialiy transient, and energy loss rate is explicitly time-dependent.
\end{abstract}

1. Introduction

Having no possibility to ionize and to excite molecules, subexcitation particles (electrons, positrons, muons) lose their energy by exciting molecular vibrations, by transferring to the medium a definite quanta of energy. In polar media there is an additional way of energy losses: orientation of the dipole molecules. Fortunately, these processes could be treated in the frame of classical electrodynamics, because during its slowing down, the particle loses its energy $W$ quasi-continuously, by small portions in comparison with $w$, exciting vibrations and ordering slightly dipoles.

Since the paper of Tachiya and Sano (i) devoted to the theoretical consideration of the Debye energy losses of finite size particles, the problem seemed to have reached to its final solution. Nevertheless, recently was found (2) a puzzling disagreement between their formula and the zwanzig expression (3) for dielectric friction force in the case of slow moving particle. In (2) were pointed out two causes which have lead to erroneous formulas in (1), and developed another approach for calculation of the energy loss rate.

Being an exactly solvable model, the problem of the Debye energy losses of the charged finite size particle now has more pedagogical rather then practical interest, because, as it was noted by different authors, the processes of excitation of intra- and intermolecular vibrations give the main contribution to the slowing down of the charged particle. Previous considerations of this problem have three drawbacks which we will try to overcome in this work:

1. Taking into account excitation of vibrations into the total loss rate $-\dot{W}$ of the particle, one usually starts $(4,5)$ from the known expression for $-\dot{W}$ in the case of stationary moving particle

$$
-\dot{W}=-\frac{I}{2 \pi} \int_{0}^{\infty} \operatorname{Im} \frac{\omega}{\varepsilon(\omega)} \cdot J(\omega) \mathrm{d} \omega,
$$


where $J(\omega)$ is a spectral function which depends on the type of motion of the particle (2), $\varepsilon(\omega)$ is the dielectric susceptibility. Then, the function $\operatorname{Im} \frac{\omega}{\varepsilon(\omega)}$ is independently summed from the inverse Debye part of dielectric susceptibility and resonance-like terms which correspond to vibration excitations. Really, such a procedure is not exact because $1 / \varepsilon(\omega)$ is not an additive quantity, while $\varepsilon(\omega)$ (or polarizability) is.

2 . Ionization process and subsequent slowing down of the electron is essentially transient: thermalization time $t_{t h}$ could be comparable with the Debye dipole relaxation time $\varepsilon_{\infty} \tau / \varepsilon_{0} \approx 200$ fs (see section 3). Therefore, starting the calculations from eq.1, which represents stationary motion of the particle seems questionable.

3. Obviously knocked out electron interacts strongly with parent ion at small distances between them. This should also be taken into account as well as time dependent character of the screening at the considered time scale.

The aim of the present paper is a consideration of the slowing down process of the light charged particle of a finite size in polar medium with time dispersion. As applications, we keep in mind thermalization process of the knocked out subexcitation secondary electrons. Our approach allows to estimate their time and length of thermalization. These values are of interest in many problems in radiochemistry and radiobiology.

2. Dynamics of the slowing down particle

In dispersive media, it is impossible to give a reasonable definition for the energy of electro-magnetic field as a thermodynamic quantity. Nevertheless an expression for the electro-magnetic flow $\mathrm{S}$ $=\frac{C}{4 \pi}[\mathbf{E H}]$ holds in dispersive media (6). The following relationship for the energy conservation law in differential form follows directly from the Maxiell's equations

div $S=-\frac{1}{4 \pi}\left(E \frac{\partial D}{\partial t}+H \frac{\partial B}{\partial t}\right)-\sum \rho_{i} \vec{v}_{i} E$.

Here $E(r, t)$ and $D(r, t)$ are the electric field and the displacement at point $r$ and time $t$, produced by the particles. $\rho_{i}$ describes charge distribution of the i-th particle, which has the velocity $\vec{w}_{i}$.

Integration of div $S$ over the whole space gives the variation per a unit of time of energy of the field, the medium and the kinetic energy $W$ of the particles, and because these entities compose an isolated system, $\int \mathrm{d}^{3} r \mathrm{div} S=0$. Hence

$$
-\dot{W}=\frac{1}{4 \pi} \int d^{3} r E(r, t) \frac{\partial}{\partial t} D(r, t), \quad \dot{W}=\sum \int d^{3} r \rho_{i} \vec{v}_{i} E .
$$

We omitted here the magnetic term, which is very small for the particles moving with velocities much less than that of light.

Let the charge distribution of the particles be constant inside a sphere with the radius a. As applications, we identify a with the radius of the wigner-Seitz cell.

In eq. $3 D(r, t)$ is the sum of the displacement $D_{i}(r)$ of the ion, which we assume to be unmovable, and $D_{e}(r-x(t))$ is the displacement of the electron placed at $x(t)$ :

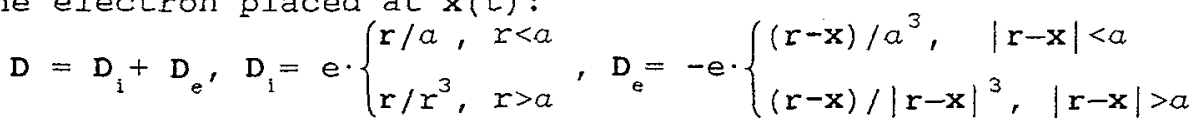

Here $e$ is the positive elementary charge.

The nature of the dielectric losses relates to the polar medium 
which can not adjust rapidly enough to the instantaneous position of the particle. The delayed reorientation of the dipoles results in an electrical drag force on a moving particle. Such a retarded response of the polarization $P(r, t)$ may be described in terms of the memory function $\gamma(t)$

$$
P(r, t)=\frac{1}{4 \pi} \int_{0}^{t} d t_{1} \gamma\left(t_{1}\right) D\left(r, t-t_{1}\right) \text {. }
$$

If we take into account excitation of vibrations, this equation holds as well. Fourier inversion in frequency leads to the relationship between $\gamma(t)$ and the reciprocal dielectric susceptibility:

$$
\int_{0}^{\infty} e^{i \omega t} \gamma(t) d t=1-\frac{1}{\varepsilon(\omega)} \text {. }
$$

Above formulas assume that $D(r, t)=E(r, t)+4 \pi P(r, t)$ in the time representation and

$$
D(r, \omega)=\varepsilon(\omega) \cdot E(r, \omega)=E(r, \omega)+4 \pi P(r, \omega)
$$

in the Fourier transform. Using eq. 5 we can rewrite eq. 3 as

$$
\begin{aligned}
-\dot{W}= & \frac{1}{4 \pi} \int d^{3} r\left(D_{i}(r)-4 \pi P(r, t)\right) \frac{\partial}{\partial t} D_{e}(r-x(t))= \\
= & \frac{1}{4 \pi}\left[\left(1-\int_{0}^{t} \gamma\left(t_{1}\right) d t_{1}\right) \cdot \int d^{3} r D_{i}(r) \frac{\partial}{\partial t} D_{e}(r-x(t))-\right. \\
& -\int_{0}^{t} \gamma\left(t_{1}\right) d t_{1} \cdot \int d^{3} r D_{e}\left(r-x\left(t-t_{1}\right)\right) \frac{\partial}{\partial t} D_{e}(r-x(t)) .
\end{aligned}
$$

Here $x(t)=\int_{0}^{t} \vec{w} t_{1}, v(t)=\left(2 \mathrm{w} / \mathrm{m}_{e}\right)^{1 / 2}$ is the velocity of the particle and $m_{e}$ is its mass. Calculations of the integrais involved gives

$$
\begin{aligned}
& \int d^{3} r D_{e}(r) \frac{\partial}{\partial t} D_{e}(r-x(t))= \\
& =v \int d^{3} r D_{e}(r) \frac{\partial}{\partial x} D_{e}(r-x)=-v \frac{4 \pi e^{2}}{a^{2}} \cdot \begin{cases}x / a, & x<a \\
(a / x)^{2}, & x>a .\end{cases}
\end{aligned}
$$

Here we carry out an integration over the whole space, including the volume of the particles. If we would like to calculate energy losses outside the moving particle only, in eq.9 we should exclude the volume of the particle around its final position at $r-x(t)$. In this case the last integral is by a factor of $2 / 3$ smaller in accordance with (3, eq.16).

Further calculations correspond to the straightline removal of the knocked out electron from the parent ion, i.e. $x(t)=\int_{0}\left(t_{1}\right) d t_{1}$. It implies that there exists unambiguous relation between $w$, $x$ and $t$, which could be obtained inserting eq.9 into eq. 8 (we assume here that $t \geq a / v$ and $x(0) \geq a)$

$$
\begin{aligned}
& -\dot{W}=\frac{e^{2} v}{a^{2}}\left[\left(1-j \gamma\left(t_{1}\right) d t_{1}\right) \cdot\left(\frac{a}{x(t)}\right)^{2}+\right. \\
& \left.+\int_{0}^{a / v} \gamma\left(t_{1}\right) \frac{x(t)-x\left(t-t_{1}\right)}{a} d t_{1}+\int_{a / v}^{t} \gamma\left(t_{1}\right)\left(\frac{a}{x(t)-x\left(t-t_{1}\right)}\right)^{2} a t_{1}\right]
\end{aligned}
$$

Assuming for simplicity $x(t)-x\left(t-t_{1}\right)=v(t) \cdot t_{1}$, finally we get

$$
-\dot{W}=\frac{e^{2} v}{a^{2}}\left[\left(\underset{0}{t} r\left(t_{1}\right) d t_{1}\right) \cdot\left(\frac{a}{x(t)}\right)^{2}+\right.
$$




$$
\left.+\frac{v(t)}{a} \int_{0}^{a / v} t_{1} \gamma\left(t_{1}\right) d t_{1}+\left(\frac{a}{v(t)}\right)^{2} \int_{a / v}^{t} \frac{\gamma\left(t_{1}\right)}{t_{1}^{2}} d t_{1}\right] .
$$

\section{Memory function $\gamma(t)$}

Polarizability of the molecule (and consequently, dielectric susceptibility of the medium) itself represents the sum of additive contributions: electron, orientational, vibrational with respect to different types of polarization. Applying oscillator model for the description of vibrational polarization, and using standard expression accounting for Debye relaxation, we obtain the following formula for total frequency dependent dielectric susceptibility

$$
\varepsilon(\omega)=\varepsilon_{\infty}+\frac{\varepsilon_{0}-\varepsilon_{\infty}}{1-i \omega \tau}+\omega_{p}^{2} \sum_{j} \frac{f_{j}}{\omega_{0 j}^{2}-\omega^{2}-i \omega \Gamma,}, \quad \omega_{p}^{2}=\frac{4 \pi e^{2}}{\Omega m_{p}} .
$$

Here $\varepsilon_{0}$ and $\varepsilon_{\infty}$ are the static and high frequency Debye dielectric constants and $\tau$ is the Debye relaxation time. $f_{j}$ is the oscillator strength characterizing the intensity of $j-t h$ vibration, $\Gamma_{j}$, its dumping and $\omega_{0 j}$ is its resonance frequency. $\Omega$ is the volume per one molecule, $m_{p}$ is the proton mass. $\omega_{p}=2.41 \cdot 10^{14} \mathrm{~s}^{-1}$ or $1277 \mathrm{~cm}^{-1}$.

Taking the Fourier transform from eq. 6 , we can express $\gamma(t)$

$$
\gamma(t)=\frac{1}{2 \pi} \int_{-\infty}^{+\infty} e^{-i \omega t}\left(1-\frac{1}{\varepsilon(\omega)}\right) d \omega .
$$

First of all we extract from $1-\frac{1}{\varepsilon(\omega)}$ the frequency independent part $1-\frac{1}{\varepsilon_{\infty}}$, which describes the instantaneous response because of the electron polarization:

$$
1-\frac{1}{\varepsilon(\omega)}=1-\frac{1}{\varepsilon_{\infty}}+\frac{1}{\varepsilon_{\infty}}\left(1-\frac{1}{1+\frac{\varepsilon-1}{1-i \omega \tau}+\sum_{j} \frac{\Omega_{j}^{2}}{\omega_{0 j}^{2}-\omega^{2}-i \omega \Gamma_{j}}}\right) \text {. }
$$

Here $\Omega_{j}^{2}=\frac{\omega_{p}^{2} f}{\varepsilon_{\infty}}$ and $\varepsilon=\varepsilon_{0} / \varepsilon_{\infty}$. Term $1-\frac{1}{\varepsilon_{\infty}}$ gives a $\delta$-type contribution into $r(t)$. Now we have to find the poles of the last term $\frac{1}{\varepsilon}-\frac{1}{\varepsilon(\omega)}$ in eq.14. Without vibrational modes, pole responsible for Debye relaxation, is in the complex plane at

$$
\omega=-i \frac{\varepsilon_{0}}{\varepsilon_{\infty} \tau}=-\frac{i}{\tau_{r}}, \quad \tau_{r}=\tau / \varepsilon, \quad \tau=0.85 \cdot 10^{-11} \mathrm{~s} \text {, in water. }
$$

As a rule this frequency is essentially smaller than resonance vibrational frequencies. Therefore from eq. 14 we obtain the shift of the Debye pole because of the presence of vibrational excitations in the following form

$$
\omega=-i \omega_{D}, \quad \omega_{D}=\frac{1+\varphi / \varepsilon}{(1+\varphi) \tau_{j}}, \varphi=\sum_{j} \Omega_{j}^{2} / \omega_{0 j}^{2} .
$$

Neglecting the overlapping of the different vibrational resonances in $\varepsilon(\omega)$, the positions of the poles are defined by equations

$$
\tilde{\omega}= \pm \tilde{\omega}_{0 j}, \tilde{\omega}=\omega+i \Gamma_{\mathrm{j}} / 2, \quad \tilde{\omega}_{0 \mathrm{j}}^{2}=\omega_{0 \mathrm{j}}^{2}+\Omega_{\mathrm{j}}^{2}\left(\Gamma_{\mathrm{j}} / 2\right)^{2} .
$$

calculating the integral in eq.13 using the subtractions technique, 
we find

$$
\begin{aligned}
\gamma(t) & =\left(1-\frac{1}{\varepsilon_{\infty}}\right) \delta(t-0)+\frac{\varepsilon-1}{\varepsilon_{\infty} \tau} \frac{1-\varphi}{1+\varphi} \exp \left(-\omega_{D} t\right)+ \\
& +\sum_{j} \frac{\Omega_{j}^{2}}{\varepsilon_{\infty} \tilde{\omega}_{0 j}} \cdot \exp \left(-\Gamma_{j} t / 2\right) \cdot\left(\sin \tilde{\omega}_{0 j} t+\frac{\varepsilon-1}{\tilde{\omega}_{0 j} \tau} \cos \tilde{\omega}_{0 j} t\right)
\end{aligned}
$$

If we integrate this expression over $t$ from 0 to $\infty$, in leading order on $\varphi$ and in the limit $\tilde{\omega}_{0 j} \gg \Gamma_{3} / 2$, we reproduce eq. $\sigma$ for $\omega=0$

$$
\int_{0}^{\infty} \gamma(t) d t=1-\frac{1}{\varepsilon_{0}+\sum_{j} \omega_{p}^{2} f_{j} / \omega_{0 j}^{2}}=1-\frac{1}{\varepsilon_{s}}, \quad \varepsilon_{0}=\varepsilon_{s}-\sum_{j} \frac{\omega_{p}^{2} f_{j}}{\omega_{0 j}^{2}} .
$$

Here $\varepsilon_{s}$ is the static dielectric constant (for water $\varepsilon_{s}=78$ ). It is necessary to note that the number of resonance terms in eq.17 strongly depends from the energy of the particle. We have to multiply each $f_{j}$ on $\vartheta\left(W-n \omega_{0 j}\right)(\vartheta(z>0)=1, \vartheta(z<0)=0)$. It implies obvious restriction: the particle with the energy $w$ could not excite vibrations with the energy $\hbar \omega_{0 j}$, if $W<n \omega_{0 j}$.

Substituting eq.17 into eq.11 we obtain the final expression for $-\dot{W}$. Integrals in eq. 11 could be expressed through the special functions $E_{1}(z), S i(z)$ and $C i(z)$.

To find the values of parameters $f_{j}, \tilde{\omega}_{0 j}, \Gamma_{j}$ and $\varepsilon_{0}$ let us express -Im $1 / \varepsilon(\omega)$ from eq.12 in the vicinity of resonance vibrational maxima on the real axes

$$
\begin{aligned}
& -\operatorname{Im} 1 /\left.\varepsilon(\omega)\right|_{\omega \approx \omega_{1 j}}=\frac{\Omega_{j}^{2}}{4 \varepsilon_{\infty} \omega_{1 j}} \frac{\Gamma_{j}}{\left(\omega_{1 j}-\omega\right)^{2}+\left(\Gamma_{j} / 2\right)^{2}}, \\
& \omega_{1 j}^{2}=\omega_{0 j}^{2}+\Omega_{j}^{2}=\tilde{\omega}_{0 j}^{2}+\left(\Gamma_{j} / 2\right)^{2},
\end{aligned}
$$

and fit the experimental data $(7,8)$ for $-\operatorname{Im} 1 / \varepsilon(\omega)$ in water at room temperature, fig.1. As a result we obtain $\left(\varepsilon_{\infty}=1.8\right)$

$\begin{array}{cccccc}\omega_{1 j^{\prime}}, \mathrm{cm}^{-1} & \tilde{\omega}_{0 j^{\prime}} \mathrm{cm}^{-1} & \omega_{0 j}, \mathrm{~cm}^{-1} & \hbar \omega_{0 j}, \mathrm{eV} & f_{j} & \Gamma_{j},^{\mathrm{cm}^{-1}} \\ 220 & 201 & 198 & 0.025 & 0.01 & 160-180 \\ 770 & 728 & 640 & 0.079 & 0.2 & 400-500 \\ 1690 & 1684 & 1677 & 0.21 & 0.05 & 110 \\ 3500 & 3494 & 3400 & 0.42 & 0.7 & 350-390\end{array}$

\section{Resuits}

To visualize the influence of vibrational resonances on the Debye losses let us present the relationship for $-\dot{W}$ in the limit of stationary moving particle: $t \rightarrow \infty$, but $v=$ const. In this case from eq. 11 and in the particular interesting limit of $v \tau_{r} / a \gg 1$ we obtain

$$
-\dot{W}=\frac{e^{2}}{a \tau_{r}}\left[\frac{3}{2}\left(\frac{1}{\varepsilon_{\infty}}-\frac{1}{\varepsilon_{0}}\right) \frac{1}{1-\varphi}+\frac{a \tau_{r}}{\varepsilon_{\infty} v} \sum_{j} \Omega_{j}^{2} \vartheta\left(W-\hbar \omega_{0 j}\right) \text { In } \frac{v}{a \omega_{1 j}}\right] .
$$

Multiplier $\frac{1}{1-\varphi}$ presents the mutual "interference" of the Debye

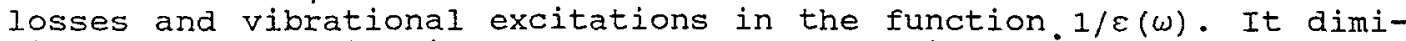
nishes the contribution of the Debye losses into $-\dot{W}$. Eq. 20 could be directly derived from eq.1, using eq.14 for $1 / \varepsilon(\omega)$.

The solution of eq.11 is presented on fig.2. It describes ther- 
Fig. I Imaginary part of the inverse dielectric susceptibility of liquid water at room temperature. Recalculated from $(7,8)$.

Fig.2 slowing down of the knocked out subexcitation electron in water at room temperature. Straightline removal from the parent ion is assumed. $x(t)$ is the coordinate of: the electron, $a=1.93 \AA$, the radius of Wignerseitz cell. $W(t)$, the kinetic energy of the electron, and $-\dot{W}$, its energy loss rate.

Fig.3 Thermalization distance in the units of wigner-seitz radius $a$, and thermalization time in $f s$ vs initial excess kinetic energy $W_{\infty}$ 。
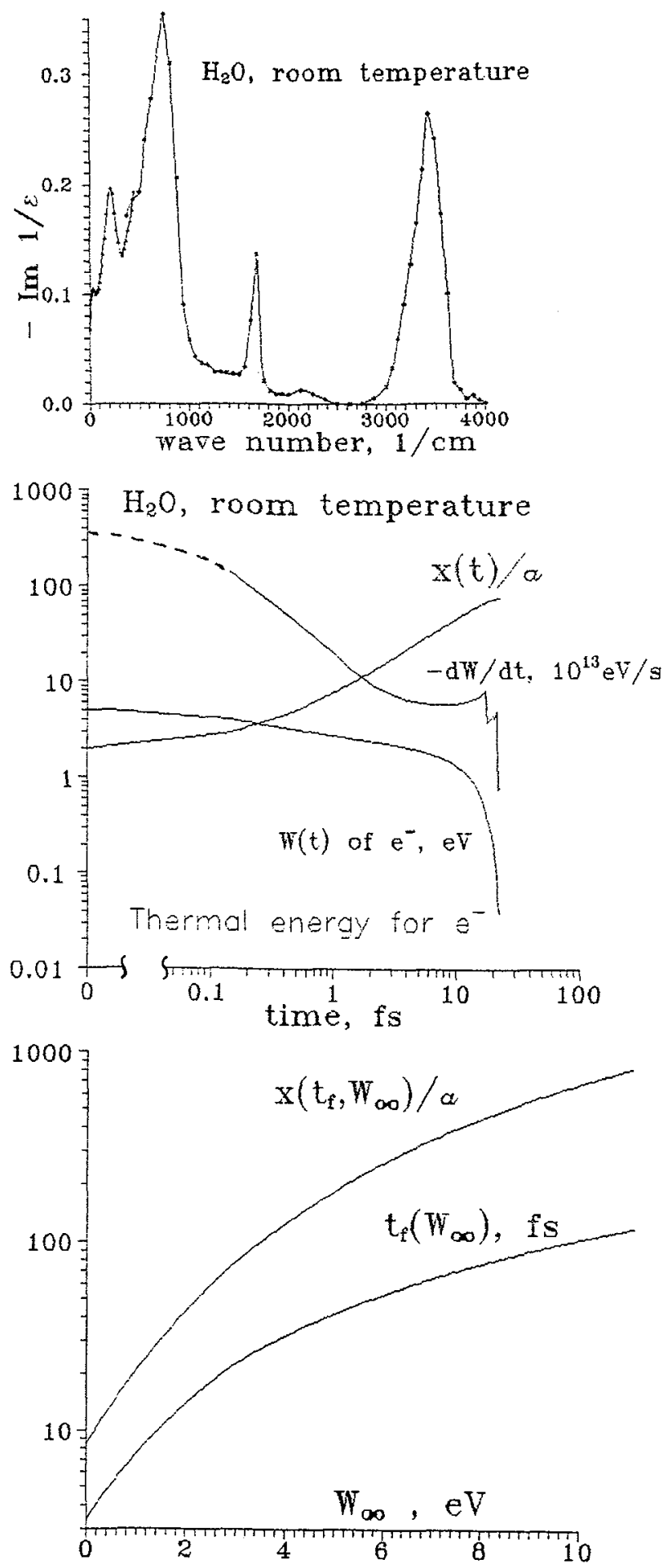
malization of the subexcitation secondary electron in water at room temperature. We assume that at $t=0$ electron has an initial kinetic energy $W(t=0)=\frac{e^{2}}{\varepsilon_{\infty} x(t=0)}+W_{\infty}$ and starts its motion at $x(t=0)$. Really particular choice of $x(t=0)$ is not very important for our calculations, because corresponding change of initial kinetic energy will be compensated because of the interaction with the parent ion. $W_{\infty}$ is the excess (over ionization potential) initial kinetic energy of the electron. Roughly speaking, if the primary particle transfer to the secondary electron the energy $I_{L}+W_{\infty}$ ( $I_{L}$ is the ionization potential in liquid) then, passing the distance $\leq 10 a$, as it is seen from fig.2, the particle loses the first part, $I_{L}$, because of the interaction with the ion. On these distances the contribution of vibrational excitations and Debye losses into the slowing down process is small. At $x(t) \geq 10 a$ thermalization process is governed by the energy dissipation to the medium and at time $t_{f}\left(W_{\infty}\right)$ and distance $x\left(t_{f}, w_{\infty}\right)$ the kinetic energy of the electron decreases down to thermal.

To obtain thermalization time $t_{t h}$ and distance $\ell_{t h}$, it is necessary to average $t_{f}\left(W_{\infty}\right)$ and $x\left(t_{f}, w_{\infty}\right)$ over $W_{\infty}$. corresponding distribution function is known, and for knocked out electrons with $W_{\infty}<50 \mathrm{eV}$
has the form

$$
f\left(W_{\infty}\right) \propto\left[I_{L} /\left(I_{L}+W_{\infty}\right)\right]^{4.5}
$$

In water $I_{L}=8.7-8.9 \mathrm{eV}(9)$. On fig.3 are shown the dependencies $t_{f}\left(W_{\infty}\right)$ and $x\left(t_{f}, w_{\infty}\right)$, which have to be weighted by $f\left(w_{\infty}\right)$. We consider here slowing down of subexcitation electrons only. So we did not take into account possible subsequent ionizations by secondary electrons. Therefore $W_{\infty}$ has to be less then $I_{L}$. Averaging $t_{f}\left(W_{\infty}\right)$ and $x\left(t_{f}, W_{\infty}\right)$ with the weight $f\left(W_{\infty}\right)$ within the energy interval $0<W_{\infty}<I_{L}$, we finally obtain $t_{t h}=15-18$ fs and $\ell_{t h}=50-60 a \approx 100-120 \dot{A}$.

/1/ TACHIYA, M, SANO,H, J.Chem.Phys. 67(11), (1978) 5111

/2/ ZWANZIG, R, Phys.Rev. 38(7), (1963) 1603

/3/ STEPANOV, S, V, Preprint ITEP N79, MOSCOW, 1992

/4/ GARIN, M, BYAKOV, V, M, High Energy Chem. 22(3), (1988) 195

/5/ MAGEE, J, L, HELMAN, W, P, J.Chem. Phys. 66(I), (1977) 310

/6/ LANDAU, L, D, LIFSHITS, E, M, Electrodynamics of Continues Media, Pergamon, New York, 1960

/7/ QUERRY,M,R, TYLER, I, I J.Chem.Phys. 72(4), (1980) 2495

/8/ AFSAR, M, N, HASTED, J, B, J.Opt.SOC.Am. 67(7), (1977) 902

$19 /$ BYAKOV, V, M, NICHIPOROV, F, G, Intratrack Chemical Processes (in Russian), Moscow, Energoatomizdat, 1985

/10/ BYAKov, V, M, STEPANov, S,V, Rad.Phys.Chem. 41(3), (1993), 559 